\title{
Mir-758-5p Suppresses Glioblastoma Proliferation, Migration and Invasion by Targeting ZBTB20
}

\author{
Ji Liu Jian Jiang Xiaobo Hui Weijie Wang Dazhao Fang Lianshu Ding \\ Department of Neurosurgery, the Affiliated Huaian No.1 People's Hospital of Nanjing Medical University, \\ Huai'an, Jiangsu Province, China
}

\section{Key Words}

Mir-758-5p • Proliferation • Metastasis • ZBTB20 • Glioblastoma

\begin{abstract}
Background/Aims: To determine the cellular functions and clinical significance of micro-758$5 p$ (miR-758-5p) in glioblastoma (GBM) by targeting zinc finger and BTB domain-containing protein 20 (ZBTB20). Methods: Fifty-five paired GBM tissues and adjacent normal tissues, GBM cell lines (U118, LN-299, H4, A172, U87-MG, and U251), and normal human astrocyte cell line (HEB) were used. miR-758-5p mimics, ZBTB20 siRNA, and pcDNA3.1-ZBTB20 were transiently transduced into cancer cells independently or together. $\mathrm{qRT}$-PCR was conducted to analyze the expression of miR-758-5p and ZBTB20. Luciferase reporter assays were performed to determine the effect of miR-758-5p on ZBTB20. Western blot was applied to measure the expression of ZBTB20, PCNA, and cleaved caspase3. Cell Counting Kit-8 (CCK8), colony formation, FACS, and Transwell assays were carried out to detect cellular proliferation, apoptosis, migration, and invasion. Xenograft experiments were implemented to evaluate tumor growth and metastasis in vivo. Results: miR-758-5p was significantly downregulated in GBM tissues and cell lines compared with that in adjacent normal tissues and HEB cells. miR-758-5p overexpression inhibited the proliferation, migration, and invasion of GBM cells and induced apoptosis by regulating the ZBTB20 expression. Pearson correlation analysis also confirmed that miR758-5p was inversely correlated with ZBTB20 in GBM tissues. miR-758-5p suppressed tumor growth and metastasis in vivo. The restored ZBTB20 expression partially rescued the miR758-5p-induced inhibition of GBM cell proliferation, migration, and invasion. Kaplan-Meier curve analysis revealed that a high miR-758-5p expression indicated an enhanced prognosis of patients with GBM. Conclusion: miR-758-5p suppressed GBM progression by targeting ZBTB20. The miR-758-5p/ZBTB20 axis might be a promising therapeutic target for GBM treatment.
\end{abstract}

\section{Introduction}

(C) 2018 The Author(s)

Published by S. Karger AG, Basel

As a lethal malignant tumor, glioblastoma (GBM) is the most common primary brain tumor [1]. Although GBM therapy has remarkably improved, early diagnosis remains 
difficult. As the most commonly used method, surgical resection followed by concomitant chemoradiation for GBM treatment is inefficient $[2,3]$. The median survival of 14.6 months is low $[4,5]$. As such, the mechanisms of the progression of this complicated disease should be understood, and new treatment methods should be developed.

MicroRNAs (miRNAs) are a new class of highly conserved non-protein-encoding small molecules that regulate gene expression through an miR-RISC complex [6]. With the binding of miR-RISC complex to the 3'untranslated region (UTR) of target mRNAs, target gene expression is usually suppressed [7]. miRNAs play an important role in a wide range of biological processes, including cell proliferation, apoptosis, and cancer progression [810]. Human miRNA genes are frequently found co-localized with genomic regions involved in cancer [11]. These genes also have altered expression in different types of human cancers. Therefore, miRNAs may serve as biomarkers for cancer diagnosis, prognosis, and pathogenesis $[12,13]$.

Studies have shown the functions of miRNAs in GBM. More than 300 miRNAs are differently regulated in GBM compared with that in normal tissues [14], and their expression is usually correlated with various glioma stages $[15,16]$. miRNAs regulate the proliferation, differentiation, and apoptosis of GBM [17-19]. To further study the role of miRNAs in GBM progression, we conducted an unbiased analysis of the GBM database and found that miR758-5p was downregulated in GBM tissues. miR-758-5p participates in cholesterol uptake [20] and possibly functions in cancer progression [21,22], but its role in GBM has yet to be investigated. In this study, we found that miR-758-5p acted as a GBM suppressor gene, suppressed GBM cell proliferation, invasion, and migration, and induced GBM apoptosis. miR-758-5p mechanically bound to the 3'UTR of ZBTB20, inhibited ZBTB20 expression, and further suppressed GBM progression.

\section{Materials and Methods}

\section{Human samples}

Paraffin-embedded tumor specimens from 55 cases were surgically collected from The Affiliated Huaian No.1 People's Hospital of Nanjing Medical University. The diagnosis of GBM in these cases was confirmed through a pathological examination. All of the patients in this study did not receive chemotherapy or radiotherapy before they underwent surgical excision. In addition, 55 cases of normal brain tissues (nontumor tissues) were obtained as a control. All of the patients provided a signed informed consent. Our study was approved by the ethics committee of The Affiliated Huaian No.1 People's Hospital of Nanjing Medical University.

\section{Cell culture}

HEB was purchased from the Guangzhou Institute of Biomedicine and Health, Chinese Academy of Sciences. U118, LN-299, H4, A172, and U87-MG were procured from the American Type Culture Collection (ATCC, USA). U251 cell was obtained from Cell Lines Service (CLS, Germany). All of the cells were maintained in DMEM (Gibco, Thermo Scientific, New York, NY, USA) supplemented with $10 \%$ fetal bovine serum (FBS, Gibco, Thermo Scientific, New York, NY, USA) and $1 \%$ sodium pyruvate and cultured at $37{ }^{\circ} \mathrm{C}$ in $5 \% \mathrm{CO}_{2}$. miR-758-5p mimics, negative control, and ZBTB20 siRNA were synthesized by GenePharma (Shanghai, China), and Lipofectamine 2000 (Invitrogen, USA) was used to transfect genes into cells.

\section{Quantitative real-time polymerase chain reaction ( $q R T-P C R$ )}

RNA was extracted using Trizol reagent (Thermo Fisher Scientific, USA) in accordance with the manufacturer's instructions. Total mRNA $(1 \mu \mathrm{g})$ was reverse transcribed into cDNA with SuperScript ${ }^{\mathrm{TM}}$ III First-strand Synthesis System (Thermo Fisher Scientific, USA). RT-qPCR was performed with SYBR Green Real-time PCR Master Mixes (Thermo Fisher Scientific, USA), and U6 or GAPDH served as an internal control. Our results were analyzed using $2^{-\Delta \Delta C T}$ method. 


\section{Cellular Physiology Cell Physiol Biochem 2018;48:2074-2083 \\ \begin{tabular}{l|l} 
and Biochemistry Published online: August 9, 2018 & $\begin{array}{l}\text { C) } 2018 \text { The Author(s). Published by S. Karger AG, Basel } \\
\text { www.karger.com/cpb }\end{array}$ \\
\hline
\end{tabular}}

Liu et al.: Mir-758-5p Suppresses Glioblastoma Progression

\section{Flow cytometry}

For cell cycle analysis, the cells were fixed with 70\% ethanol and resuspended in PBS. Afterward, 50 $\mu \mathrm{g} / \mathrm{ml}$ propidium iodide (PI) and $0.2 \%$ Triton X-100 were added to the cells, incubated for another $30 \mathrm{~min}$ at $4{ }^{\circ} \mathrm{C}$, and detected with a flow cytometer (BD Biosciences, NJ, USA). The distribution of cells in G0/G1, S, and G2/M phases was established using ModFit LT (Verity Software House).

For viability analyses, the cells were trypsinized, suspended in PBS, and stained with FITC Annexin V (1:500) for $15 \mathrm{~min}$ at room temperature. Before analyses were conducted, $4 \mu \mathrm{g} / \mathrm{ml}$ PI was added to the cells and analyzed with a flow cytometer.

\section{Cell Counting Kit-8 (CCK8 assay)}

CCK8 assay was conducted in accordance with the manufacturer's instructions (WST-8, Dojindi Labs, Kumamoto, Japan). In brief, A172 and U251 cells were seeded at a density of 3000 cells/well in a 96-well plate and treated with ethanol. After 24, 48, 72, and 96 h, $10 \mu \mathrm{l}$ of CCK8 solution was added to each well of the plate, and the wells were incubated for $2 \mathrm{~h}$. Absorbance at $450 \mathrm{~nm}$ was evaluated using a Multiscan MS spectrophotometer (Labsystems, Stockholm, Sweden).

\section{Colony formation assay}

In this procedure, 500 cells in the logarithmic growth phase were plated into Petri dishes with $10 \mathrm{ml}$ of the culture medium. The cells were grown for 2 weeks at $37{ }^{\circ} \mathrm{C}, 5 \% \mathrm{CO}_{2}$, and $100 \%$ humidity without changing the medium. Afterward, the cells were fixed with $4 \%$ paraformaldehyde and stained with Giemsa for $30 \mathrm{~min}$, and colonies were counted under a microscope.

\section{In vivo assays}

BALB/c nude mice were purchased from Shanghai Slac Laboratory Animal Co., Ltd. and maintained under SPF conditions. All of the animals were used in accordance with institutional guidelines, and our experiments were approved by the Use Committee for Animal Care of The Affiliated Huaian No.1 People's Hospital of Nanjing Medical University. For tumor growth in vivo, $1 \times 10^{7} \mathrm{U} 251$ cells were implanted subcutaneously in the flanks of BALB/c nude mice (six animals/each group). The mice were checked regularly for tumor size at the indicated time points. The tumor-bearing mice were sacrificed 5 weeks postimplantation, and tumor weights were measured. Cell proliferation and apoptosis in tumor tissues were analyzed through Western blot. For glioblastoma cell metastasis assays, $1 \times 10^{7} \mathrm{U} 251$ cells were resuspended in PBS and injected into the tail vein of BALB/c nude mice. All of the mice were killed with $\mathrm{CO}_{2} 25$ days after injection. Metastatic nodules in the lung tissues were quantified as previously described [23].

\section{Transwell assays}

In this procedure, $48 \mathrm{~h}$ after transfection, $1 \times 10^{4}$ cells in serum-free DMEM were plated in the upper chamber (coated without $50 \mathrm{mg} / \mathrm{l}$ Matrigel for migration and with matrigel for invasion assay), while 600 $\mu \mathrm{l}$ of the full medium was placed in the lower chamber. The remaining cells on the upper surface were removed $24 \mathrm{~h}$ after incubation, and migrating or invading cells were fixed with $4 \%$ formaldehyde and stained with $0.1 \%$ crystal violet. For each experiment, 10 fields of view were counted, and the experiments were conducted at least three times.

\section{Luciferase assays}

The 3'-UTR target sites of the predicted target gene and a mutant variant were synthesized and cloned in the pmirGLO Dual-Luciferase miRNA target expression vector. A172 and U251 cells were seeded in 96well plates and co-transfected $12 \mathrm{~h}$ later with plasmids $(0.2 \mu \mathrm{g})$, miR-758-5p mimics (5 pmol) or scramble sequences (5 pmol) using Lipofectamine 2000 (Invitrogen, USA). The cells were harvested $48 \mathrm{~h}$ after transfection, and firefly and renilla luciferase activities were analyzed with the Dual-Luciferase ${ }^{\mathrm{TM}}$ Reporter Assay System, according to the manufacturer's instructions (Promega, USA).

\section{Western blotting}

Cells and tumor samples were homogenized with RIPA lysis buffer, and 50ug of the whole-cell lysates were separated by SDS-polyacrylamide gels and transferred to nitrocellulose membranes. The membrane was then blocked with $5 \%$ non-fat dry milk in TBST for $1 \mathrm{~h}$, and then probed with indicated antibodies 
overnight at $4^{\circ} \mathrm{C}$. HRP conjugated goat anti-rabbit or anti-mouse secondary antibodies were cultured for another hour, and enhanced chemiluminescence reagents (ECL, Amersham Bioscience) were used to visualize the bands. ACTB was used as a loading control.

\section{Statistical analyses}

Statistical analysis was performed using the Student's t-test or one-way analysis of variance followed by Fisher's least significant difference post hoc test for multiple comparisons. Results are shown as mean \pm SD. $P<0.05$ was considered statistically significant.

\section{Results}

miR-758-5p was downregulated in GBM

All of the differentially expressed miRNAs in Gulluoglu's cohort (GSE90604) were analyzed (Fig. 1A) to explore the functions of miRNAs in GBM. Among the 21 differentially expressed miRNAs, miR-758-5p was the most significantly downregulated (Figs. 1A and B). The miR-758-5p expression in GBM tumor tissues $(n=55)$ and adjacent normal tissues ( $\mathrm{n}$ = 55) was analyzed through qRT-PCR to confirm that miR-758-5p was downregulated in GBM. Our results verified that miR-758-5p was downregulated in GBM tissues compared with that in adjacent normal tissues (Fig. 1C). The miR-758-5p expression decreased as the TNM stages of GBM tissues advanced (Fig. 1D). The miR-758-5p expression levels were also downregulated in all of the GBM cell lines compared with those in the normal control cell line (HEB; Fig. 1E). A high miR-758-5p expression was correlated with a high survival rate of patients with GBM (Fig. 1F).

These results showed that miR-758-5p was downregulated in GBM. Thus, miR-758-5p might suppress GBM progression.

Upregulation of miR758-5p suppressed GBM cell proliferation and promoted apoptosis

Altered proliferation and apoptosis are two key characters that distinguish tumor cells from normal cells [24]. In our study, miR758-5p was overexpressed in A172 and U251 GBM cell lines to examine the function of miR-758-5p in GBM proliferation and apoptosis (Fig. 2A). CCK8 assays showed that the miR-188-5p overexpression significantly inhibited GBM cell proliferation (Fig 2B). Consistent with this finding, our results revealed that the colony formation ability of the GBM cells was greatly reduced when miR-758-5p

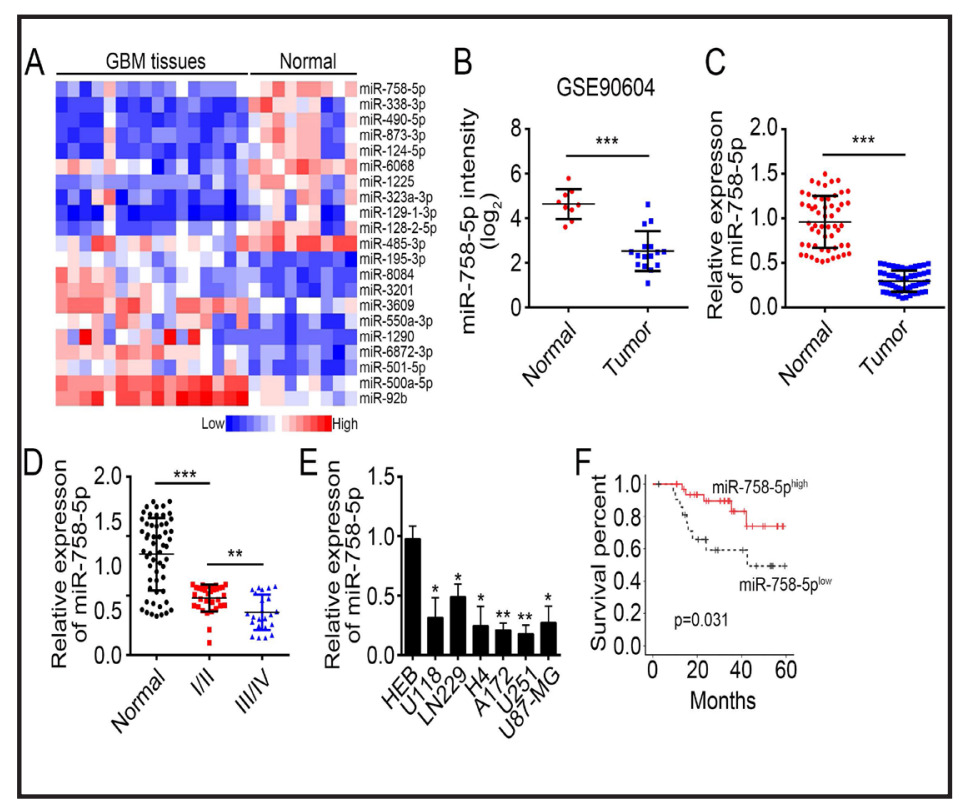

Fig. 1. miR-758-5p was downregulated in glioblastoma. (A) Heatmap of differentially expressed miRNAs in GBM tissues and normal tissues based on Gulluoglu's cohort (GSE90604). (B) miR-758-5p expression level in GBM tissues and normal tissues according to Gulluoglu's cohort (GSE90604). (C) qRT-PCR analysis of miR-758-5p expression in GBM tissues ( $n=55)$ and adjacent normal tissues ( $n=55)$. (D) Relative miR758-5p expression in different TNM stages of GBM tissues. (E) qRT-PCR analysis of the relative miR-758-5p expression in GBM cell lines (A172, U118, LN229, H4, U251, and U87-MG) and normal human astrocyte cell line (HEB). (F) Kaplan-Meier survival analysis based on the miR-758$5 \mathrm{p}$ expression in GBM tissues. ${ }^{*} \mathrm{p}<0.05{ }^{* *} \mathrm{p}<0.01$, and ${ }^{* * *} \mathrm{p}<0.001$. 
was overexpressed (Figs. 2C), indicating that miR-758-5p inhibited GBM cell proliferation. FACS was conducted to analyze the cell cycle distribution and to further evaluate the proliferation of GBM cells after miR-758-5p was overexpressed. The results showed that the miR-758-5p overexpression blocked the progression of the GBM cell cycle. When miR758-5p was overexpressed, the number of cells in the G0/G1 and G2/M phases increased, whereas the number of cells in the $S$ phase decreased (Fig. 2D). At the same time, the number of Annexin V-positive cells increased (Fig. 2E), indicating that miR-758-5p induced the apoptosis of GBM cells. Xenograft experiments also demonstrated that tumor size and volume were significantly reduced when miR-758-5p was overexpressed (Figs. $2 \mathrm{~F}$ and G). More apoptotic cells were found as indicated by more cleaved caspase 3 and more apoptotic cells (Fig. 2H). These results showed that miR-758-5p inhibited GBM progression, and miR758-5p might function as a tumor suppressor of GBM.

\section{miR-758-5p inhibited the migration and invasion of GBM cells}

To gain malignant phenotypes, tumor cells must develop their migration and invasion ability. In our study, Transwell assays were performed to analyze the migration and invasion of the GBM cells after miR-758-5p was overexpressed and to further examine the role of miR-758-5p in tumor migration and invasion. The results showed that the migration and invasion of the GBM cells were significantly reduced after miR-758-5p was overexpressed (Figs. 3A and B). The expression of mesenchymal marker genes decreased, whereas the expression of the metastasis-related genes $\alpha$-catenin and E-cadherin increased (Fig. 3C). In vivo metastasis assays also revealed few metastatic nodules in the lungs after miR-758-5p was overexpressed (Fig. 3D). These results indicated that miR-758-5p inhibited the invasion

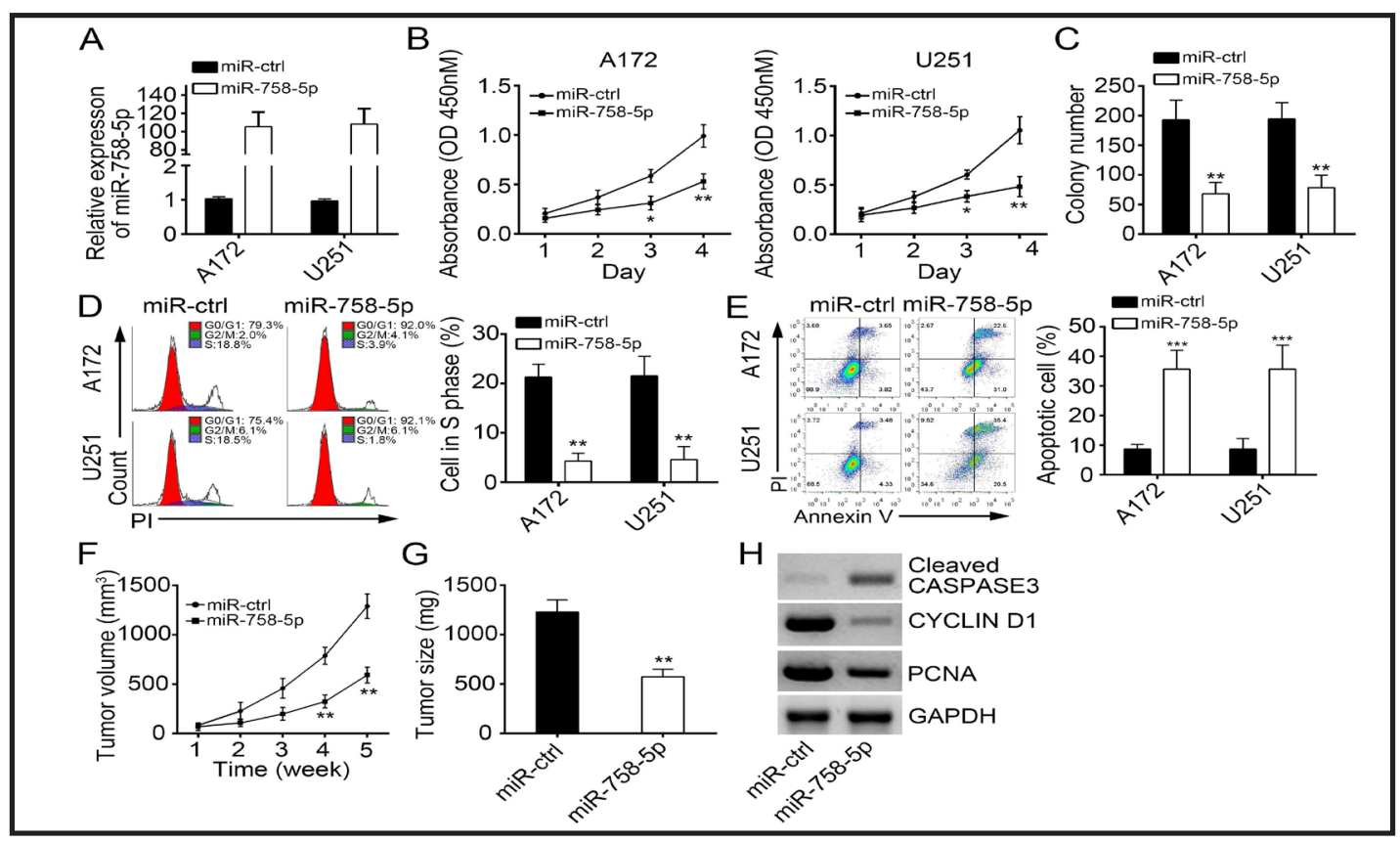

Fig. 2. miR-758-5p upregulation suppressed GBM cell proliferation and promoted apoptosis in vitro and in vivo. (A) qRT-PCR analysis of miR-758-5p expression in A172 and U251 cells transfected with miR-758-5p mimics or controls. (B) CCK8 assay of cellular proliferation by using A172 and U251 cells transfected with miR-758-5p mimics or controls. (C) Colony formation assay of cellular proliferation with A172 and U251 cells transfected with miR-758-5p mimics or controls. (D) FACS measurement of cell cycle distribution. (E) Annexin V/PI staining for the determination of apoptosis of A172 and U251 cells transfected with miR-758$5 p$ mimics or controls. (F) Tumor volumes were determined at the indicated time points. (G) Tumor sizes were analyzed at the endpoint of the xenograft experiment. $(\mathrm{H})$ Cellular proliferation and apoptosis were analyzed in the formed tumor tissues. The protein levels of cleaved CASPASE3, CYCLIN D1, and PCNA were measured by Western blot. GAPDH acted as loading control. ${ }^{*} \mathrm{p}<0.05,{ }^{* *} \mathrm{p}<0.01$ and ${ }^{* * *} \mathrm{p}<0.01$. 
and migration of GBM cells.

\section{miR-758-5p regulated ZBTB20 expression}

The gene of miRNA modules is expressed by binding to the 3'UTR of mRNA [25]. To reveal the mechanism of miR-758-5p in the regulation of GBM, we predicted the possible binding partner of miR758-5p. We found that miR-758-5p might bind to the 3'UTR of ZBTB20 (Fig. 4A). miR-758-5p mimic transfection inhibited the ZBTB20 expression (Fig. 4B). When the possible binding sites were mutated, the inhibitory effect was completely rescued (Fig. 4C). These results suggested that miR-758-5p bound to 3 'UTR and inhibited the ZBTB20 expression. qRTPCR results also showed that ZBTB20 expression was downregulated in the GBM cell lines when miR-758$5 p$ was overexpressed (Fig. 4C) but was upregulated when miR-758-5p was inhibited by a neutralizing antibody (Fig. 4D). Western blot further confirmed that miR-758-5p overexpression inhibited the ZBTB20 expression (Fig. 4E). qRT-PCR analysis also demonstrated a negative correlation between miR-758-5p and ZBTB20 expression (Fig. $4 \mathrm{~F})$. These findings were consistent with previously published data. Therefore, miR-758-5p bound to the 3'UTR of ZBTB20 and inhibited its expression.

\section{ZBTB20 promoted GBM progression}

Considering that miR-758-5p regulated the ZBTB20 expression, we further determined the function of ZBTB20 in GBM progression. Expression analysis from public databases showed that

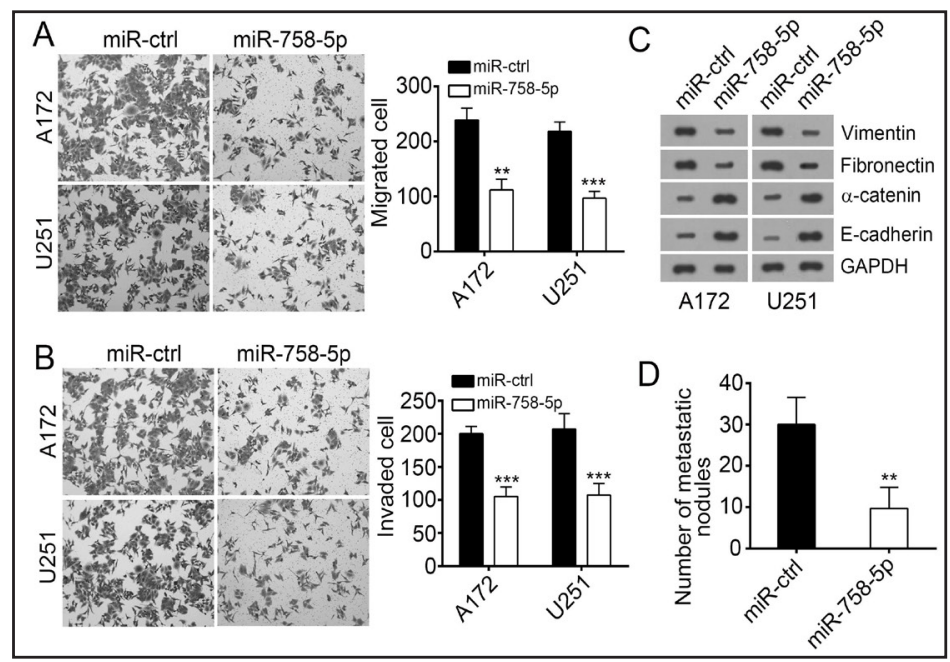

Fig. 3. miR-758-5p overexpression inhibited the migration and invasion of GBM cells in vitro and in vivo. (A and B) Cellular migration and invasion in A172 and U251 cells were determined by Transwell assay. (C) Expression levels of the epithelial markers of $\alpha$-catenin and E-cadherin and the mesenchymal markers of fibronectin and vimentin in A172 and U251 cells were verified through Western blot. (D) miR758-5p overexpression reduced the metastatic nodules in the lungs. ${ }^{* *} \mathrm{p}<0.01$ and ${ }^{* * *} \mathrm{p}<0.001$.

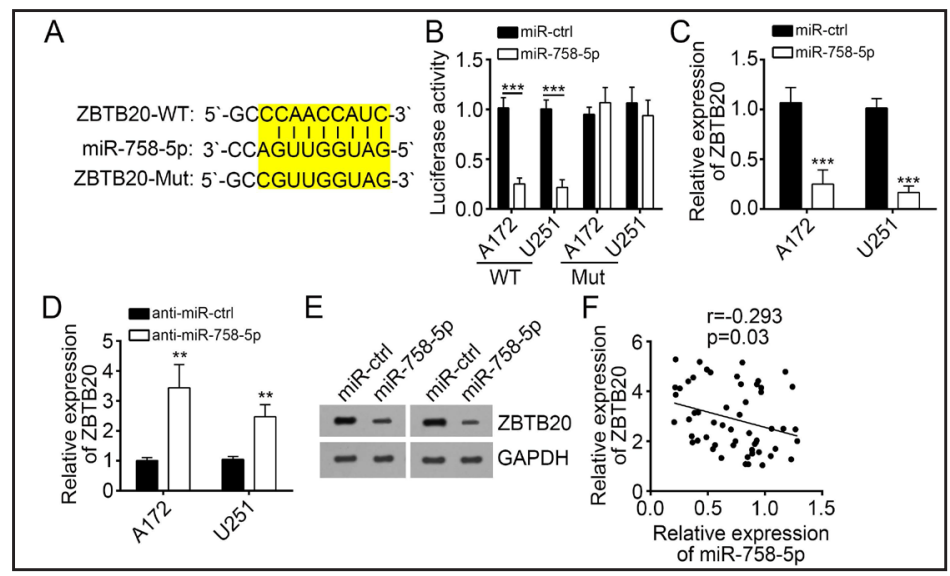

Fig. 4. ZBTB20 was a target of miR-758-5p. (A) Diagram of the potential associating site in the 3'-UTR region of ZBTB20 with miR-758-5p. (B) miR-758-5p overexpression inhibited the luciferase activity in A172 and U251 cells. (C) qRT-PCR analysis indicated that miR-758-5p overexpression inhibited the mRNA level of ZBTB20 in A172 and U251 cells. (D) miR-758-5p inhibition increased the mRNA level of ZBTB20 in A172 and U251 cells. (E) miR-758-5p overexpression inhibited the protein level of ZBTB20 in A172 and U251 cells. (F) qRT-PCR revealed a negative correlation between the expression levels of ZBTB20 and miR-758-5p in GBM tissues. ${ }^{* *} \mathrm{p}<0.01$ and ${ }^{* * *} \mathrm{p}<0.001$. 
ZBTB20 was significantly overexpressed in the GBM tissues compared with that in the normal tissues (Figs. 5A and B). The ZBTB20 expression in 9 normal tissues and 16 GBM tissues was analyzed to further confirm that ZBTB20 was overexpressed in the GBM tissues. Our results revealed that ZBTB20 was overexpressed compared with that in the normal control (Fig. 5C), and this observation was consistent with that obtained through our database analysis. The ZBTB20 expression was also higher in the GBM cell lines than in the normal control (Fig. 5D). To verify the function of ZBTB20 in GBM progression, we conducted functional assays after ZBTB20 was knocked down (Fig. 5E). We found that ZBTB20 knockdown inhibited GBM cell proliferation (Figs. 5F and G). The ZBTB20 knockdown also promoted GBM cell apoptosis (Fig. 5H). Invasion and migration assays also demonstrated that ZBTB20 knockdown inhibited GBM cell invasion and migration (Figs. 5I and J). These results suggested that ZBTB20 promoted GBM progression.

\section{miR-758-5p suppressed GBM progression through ZBTB20}

Considering that miR-758-5p suppressed GBM progression, ZBTB20 promoted GBM progression, and miR-758-5p bound to the 3'UTR of ZBTB20, we hypothesized that miR-758$5 p$ suppressed GBM progression through ZBTB20. To validate this hypothesis, we restored the ZBTB20 expression in miR-758-5p-overexpressing GBM cells (Fig. 6A) and analyzed the phenotypes. Proliferation assays revealed that ZBTB20 restoration rescued the inhibitory effect of miR-758-5p overexpression on GBM growth (Fig. 6B). Colony formation assays demonstrated that numerous colonies were generated (Fig. 6C), and the resistance of the cells to apoptosis increased (Fig. 6D). The invasion and migration abilities were also rescued after ZBTB20 expression was restored (Figs. 6E and F). These data indicated that miR-7585 p suppressed GBM progression through ZBTB20.

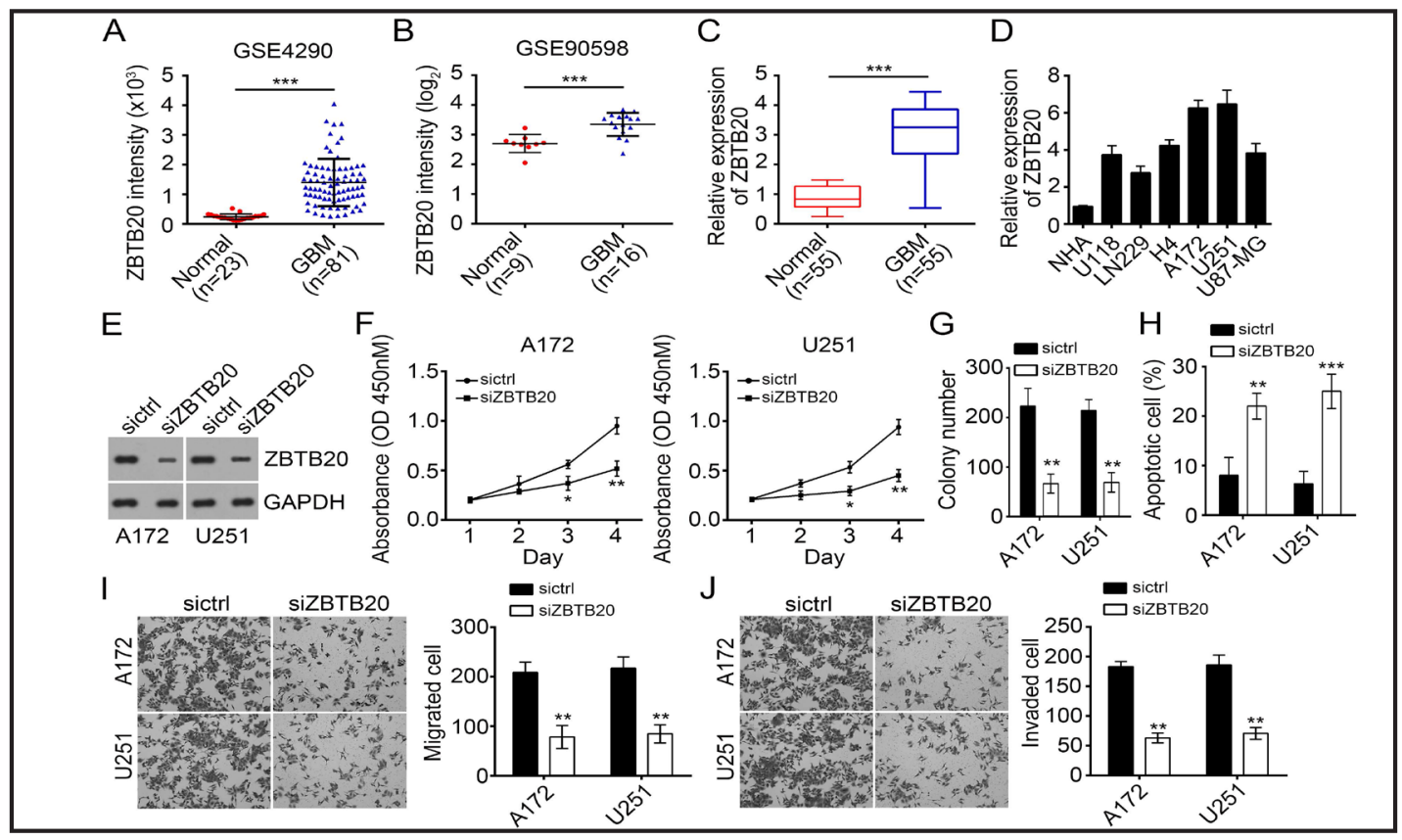

Fig. 5. ZBTB20 knockdown inhibited GBM cell proliferation, migration, and invasion. (A and B) ZBTB20 overexpression in GBM tissues compared with that in normal tissues according to Fine's cohort (GSE4290) and Gulluoglu's cohort (GSE90598). (C) qRT-PCR analysis of the relative ZBTB20 expression in 55 pairs of GBM tissues and normal tissues. (D) qRT-PCR analysis of the relative ZBTB20 expression in GBM cell lines. (E) Western blot analysis of the protein level of ZBTB20 in A172 and U251 cells transfected with siZBTB20 or control. (F and G) CCK8 and colony formation assays of the effect of ZBTB20 knockdown on cellular proliferation in A172 and U251 cells. (H) FACS analysis of the effect of ZBTB20 knockdown on cellular apoptosis in A172 and U251 cells. (I and J) Transwell assay of the migration and invasion abilities of A172 and U251 cells transfected with siZBTB2 0 or control. ${ }^{*} \mathrm{p}<0.05,{ }^{* *} \mathrm{p}<0.01$ and ${ }^{* * *} \mathrm{p}<0.01$. 


\section{Discussion}

Although studies have shown that miRNAs play an essential role in tumor progression [26], studies have yet to reveal the function of miR-758-5p in cancer. Other studies have demonstrated that miR-758-5p expression is altered in cancer, but research has yet to identify the function of miR-758-5p in cancer [21,22]. Besides, the importance of miRNAs in GBM has been emphasized by increasing evidences. For example, miR- 595 could promote GBM cell proliferation via targeting SOX7 [27]. miR-181b is reported to be implicated in GBM progression [28]. In our study, we examined the function of miR-758-5p in GBM and identified for the first time that miR-758-5p acted as a tumor suppressor gene in GBM.

Our unbiased microarray analysis demonstrated that miR-758-5p was significantly downregulated in GBM tissues, and the survival time was relatively short, indicating a possible suppressor role of miR-758-5p in GBM. Unlimited proliferation, invasion, and migration are hallmarks and characteristics of cancer [29]. Through CCK8, FACS and transwell assays, we demonstrated that miR-758-5p overexpression suppressed GBM cell proliferation, migration and invasion while promoting apoptosis, indicating a tumor suppressor role of miR-758-5p in GBM.

In a classical way of gene expression regulation, miRNAs bind to the 3'UTR of target mRNAs to regulate target gene expression [7]. Through binding partner predication, we found the possible interaction between miR-758-5p and the 3'UTR of ZBTB20 and validated their binding through mutation analysis. miR-758-5p had a negative co-expression relationship with ZBTB20, and miR-758-5p overexpression likely led to a decrease in ZBTB20 expression. Thus, ZBTB20 might be a functional target of miR-758-5p in GBM.

ZBTB20, as a zinc finger protein, has been implicated in glucose and lipid homoeostasis $[30,31]$ and in the tumor progression of hepatocellular carcinoma [32], indicating a role in cancer. In our study, ZBTB20 was overexpressed in GBM, and ZBTB20 knockdown suppressed GBM progression. This finding suggested that ZBTB20 served as a tumor progression gene. Further analysis showed that miR-758-5p suppressed GBM progression through ZBTB20 because ZBTB20 overexpression in GBM cells would rescue the malignant progression phenotypes of GBM. These results indicated that miR-758-5p and ZBTB20 axis

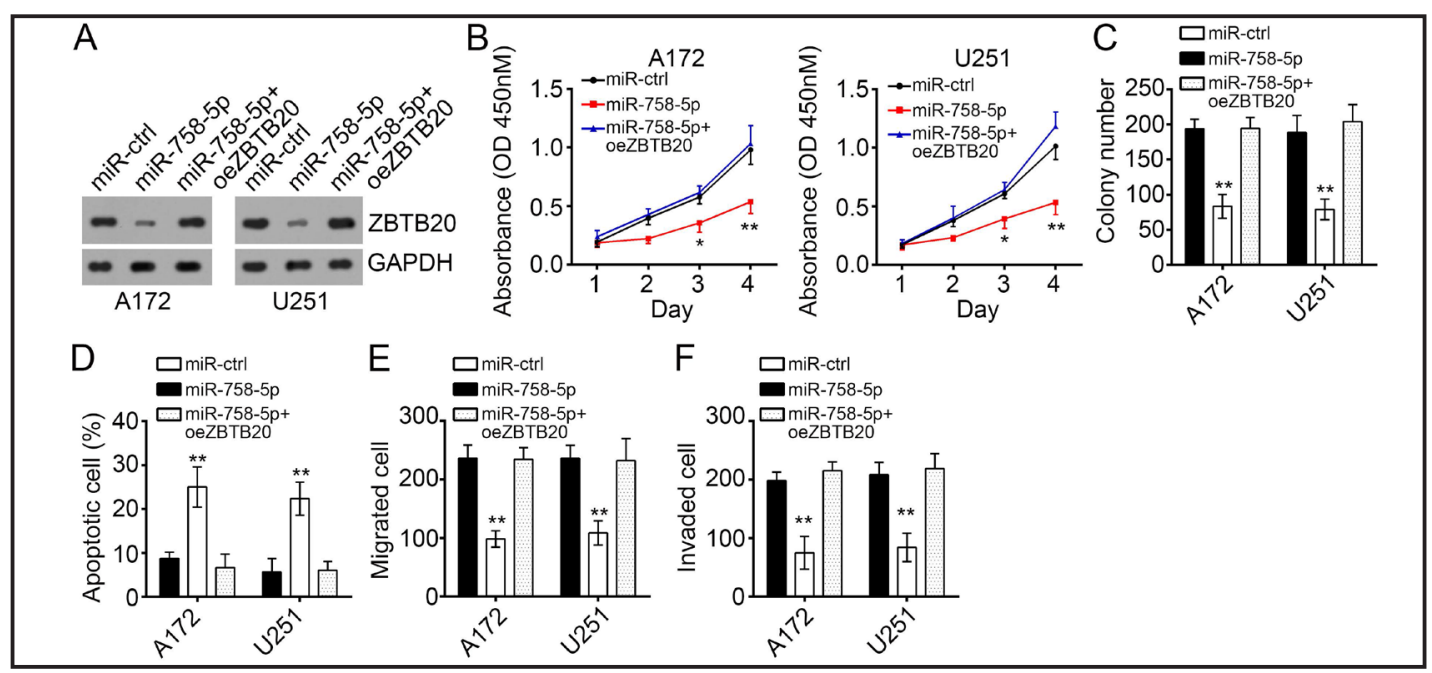

Fig. 6. ZBTB20 restoration rescued the proliferation, migration, and invasion inhibited by miR-758-5p. (A) Ectopic ZBTB20 expression restored its protein level in miR-758-5p-overexpressing A172 and U251 cells. (B and C) CCK8 and colony formation assays indicated that ZBTB20 overexpression rescued the proliferation ability of miR-758-5p-overexpressing A172 and U251 cells. (D) ZBTB20 restoration alleviated cellular apoptosis in miR-758-5p-overexpressing A172 and U251 cells. (E and F) Transwell assay revealed that the ectopic ZBTB20 expression enhanced the migration and invasion potential of miR-758-5p-overexpressing A172 and U251 cells. ${ }^{*} \mathrm{p}<0.05$ and ${ }^{* *} \mathrm{p}<0.01$. 
participated in GBM progression. miR-758-5p was downregulated in GBM, whereas ZBTB20 was upregulated in GBM.

However, there are still some limitations in our study. Firstly, the upstream regulatory mechanism of miR-758-5p expression requires to be investigated in the future. Secondly, we know there are a lot of predicted targets of miR-758-5p. Whether other target genes are also involved in the role of miR-758-5p remain largely unknown. Finally, how ZBTB20 exerts roles in GBM progression needs more experiments.

\section{Conclusion}

In this study, we demonstrated for the first time that miR-758-5p functions as a tumor suppressor gene, and the role of ZBTB20 in GBM progression. We found that miR-758-5p suppresses GBM progression, while ZBTB20 promotes GBM progression, and miR-758-5p suppresses GBM through ZBTB20. Yet, we still do not know how ZBTB20 promotes GBM progression, and this needs to be further studied.

\section{Acknowledgements}

Funding/Support: This study was supported by the "533 talent project" research foundation of Huai'an (HAA201741) and Huai'an science and Technology Development Fund (HAS07025).

\section{Disclosure Statement}

Competing interests: All authors declare that they have no conflicts of interest.

\section{References}

1 Ostrom QT, Gittleman H, Farah P, Ondracek A, Chen Y, Wolinsky Y, Stroup NE, Kruchko C, Barnholtz-Sloan JS: CBTRUS statistical report: Primary brain and central nervous system tumors diagnosed in the United States in 2006-2010. Neuro Oncol 2013;15.

-2 Khosla D: Concurrent therapy to enhance radiotherapeutic outcomes in glioblastoma. Ann Transl Med 2016;4:54.

-3 Eckert M, Klumpp L, Huber SM: Cellular Effects of the Antiepileptic Drug Valproic Acid in Glioblastoma. Cell Physiol Biochem 2017;44:1591-1605.

-4 Anderson CA, Sakamoto A, Gentile DA, Ihori N, Shibuya A, Yukawa S, Naito M, Kobayashi K: Longitudinal effects of violent video games on aggression in Japan and the United States. Pediatrics 2008;122:e10671072.

5 Tseliou M, Al-Qahtani A, Alarifi S, Alkahtani SH, Stournaras C, Sourvinos G: The Role of RhoA, RhoB and RhoC GTPases in Cell Morphology, Proliferation and Migration in Human Cytomegalovirus (HCMV) Infected Glioblastoma Cells. Cell Physiol Biochem 2016;38:94-109.

6 Meister G, Landthaler M, Patkaniowska A, Dorsett Y, Teng G, Tuschl T: Human Argonaute2 mediates RNA cleavage targeted by miRNAs and siRNAs. Mol Cell 2004;15:185-197.

7 Ambros V: The functions of animal microRNAs. Nature 2004;431:350-355.

8 Calin GA, Croce CM: MicroRNA signatures in human cancers. Nat Rev Cancer 2006;6:857-866.

-9 Esquela-Kerscher A, Slack FJ: Oncomirs - microRNAs with a role in cancer. Nat Rev Cancer 2006;6:259-269.

10 Iorio MV, Ferracin M, Liu CG, Veronese A, Spizzo R, Sabbioni S, Magri E, Pedriali M, Fabbri M, Campiglio M, Menard S, Palazzo JP, Rosenberg A, Musiani P, Volinia S, Nenci I, Calin GA, Querzoli P, Negrini M, Croce CM: MicroRNA gene expression deregulation in human breast cancer. Cancer Res 2005;65:7065-7070. 


\section{Cellular Physiology Cell Physiol Biochem 2018;48:2074-2083 \begin{tabular}{l|l|l}
\hline DOI: 10.1159/000492545 & $\begin{array}{l}\text { () 2018 The Author(s). Published by S. Karger AG, Basel } \\
\text { www.karger.com/cpb }\end{array}$
\end{tabular}

11 Calin GA, Sevignani C, Dumitru CD, Hyslop T, Noch E, Yendamuri S, Shimizu M, Rattan S, Bullrich F, Negrini M, Croce CM: Human microRNA genes are frequently located at fragile sites and genomic regions involved in cancers. Proc Natl Acad Sci U S A 2004;101:2999-3004.

12 Cummins JM, Velculescu VE: Implications of micro-RNA profiling for cancer diagnosis. Oncogene 2006;25:6220-6227.

13 Ahmed FE: Role of miRNA in carcinogenesis and biomarker selection: a methodological view. Expert Rev Mol Diagn 2007;7:569-603.

14 Moller HG, Rasmussen AP, Andersen HH, Johnsen KB, Henriksen M, Duroux M: A systematic review of microRNA in glioblastoma multiforme: micro-modulators in the mesenchymal mode of migration and invasion. Mol Neurobiol 2013;47:131-144.

15 Jiang L, Mao P, Song L, Wu J, Huang J, Lin C, Yuan J, Qu L, Cheng SY, Li J: miR-182 as a prognostic marker for glioma progression and patient survival. Am J Pathol 2010;177:29-38.

16 Sun G, Cao Y, Shi L, Sun L, Wang Y, Chen C, Wan Z, Fu L, You Y: Overexpressed miRNA-137 inhibits human glioma cells growth by targeting Rac1. Cancer Biother Radiopharm 2013;28:327-334.

-17 Lee ST, Chu K, Oh HJ, Im WS, Lim JY, Kim SK, Park CK, Jung KH, Lee SK, Kim M, Roh JK: Let-7 microRNA inhibits the proliferation of human glioblastoma cells. J Neurooncol 2011;102:19-24.

18 Malzkorn B, Wolter M, Liesenberg F, Grzendowski M, Stuhler K, Meyer HE, Reifenberger G: Identification and functional characterization of microRNAs involved in the malignant progression of gliomas. Brain Pathol 2010;20:539-550.

19 Lukiw WJ, Cui JG, Li YY, Culicchia F: Up-regulation of micro-RNA-221 (miRNA-221; chr Xp11.3) and caspase-3 accompanies down-regulation of the survivin-1 homolog BIRC1 (NAIP) in glioblastoma multiforme (GBM). J Neurooncol 2009;91:27-32.

20 Liu B, Ye B, Yang L, Zhu X, Huang G, Zhu P, Du Y, Wu J, Qin X, Chen R, Tian Y, Fan Z: Long noncoding RNA $\operatorname{lncKdm} 2 \mathrm{~b}$ is required for ILC3 maintenance by initiation of Zfp292 expression. Nat Immunol 2017;18:499508.

21 Zhang Y, Li M, Ding Y, Fan Z, Zhang J, Zhang H, Jiang B, Zhu Y: Serum MicroRNA profile in patients with colon adenomas or cancer. BMC Med Genomics 2017;10:23.

22 Chang C, Liu T, Huang Y, Qin W, Yang H, Chen J: MicroRNA-134-3p is a novel potential inhibitor of human ovarian cancer stem cells by targeting RAB27A. Gene 2017;605:99-107.

23 Jiang K, Wang YP, Wang XD, Hui XB, Ding LS, Liu J, Liu D: Fms related tyrosine kinase 1 (Flt1) functions as an oncogene and regulates glioblastoma cell metastasis by regulating sonic hedgehog signaling. Am J Cancer Res 2017;7:1164-1176.

24 Evan GI, Vousden KH: Proliferation, cell cycle and apoptosis in cancer. Nature 2001;411:342-348.

25 Lee RC, Feinbaum RL, Ambros V: The C. elegans heterochronic gene lin-4 encodes small RNAs with antisense complementarity to lin-14. Cell 1993;75:843-854.

-26 Lu J, Getz G, Miska EA, Alvarez-Saavedra E, Lamb J, Peck D, Sweet-Cordero A, Ebert BL, Mak RH, Ferrando AA, Downing JR, Jacks T, Horvitz HR, Golub TR: MicroRNA expression profiles classify human cancers. Nature 2005;435:834-838.

27 Hao Y, Zhang S, Sun S, Zhu J, Xiao Y: MiR-595 targeting regulation of SOX7 expression promoted cell proliferation of human glioblastoma. Biomed Pharmacother 2016;80:121-126.

28 Xu RX, Liu RY, Wu CM, Zhao YS, Li Y, Yao YQ Xu YH: DNA damage-induced NF-kappaB activation in human glioblastoma cells promotes miR-181b expression and cell proliferation. Cell Physiol Biochem 2015;35:913-925.

-29 Hanahan D, Weinberg RA: Hallmarks of cancer: the next generation. Cell 2011;144:646-674.

-30 Sutherland AP, Zhang H, Zhang Y, Michaud M, Xie Z, Patti ME, Grusby MJ, Zhang WJ: Zinc finger protein Zbtb20 is essential for postnatal survival and glucose homeostasis. Mol Cell Biol 2009;29:2804-2815.

-31 Liu G, Zhou L, Zhang H, Chen R, Zhang Y, Li L, Lu JY, Jiang H, Liu D, Qi S, Jiang YM, Yin K, Xie Z, Shi Y, Liu Y, Cao X, Chen YX, Zou D, Zhang WJ: Regulation of hepatic lipogenesis by the zinc finger protein Zbtb20. Nat Commun 2017;8:14824.

-32 Kan H, Huang Y, Li X, Liu D, Chen J, Shu M: Zinc finger protein ZBTB20 is an independent prognostic marker and promotes tumor growth of human hepatocellular carcinoma by repressing FoxO1. Oncotarget 2016;7:14336-14349. 